\title{
Motivación intrínseca y rendimiento académico de ingresantes 2016 - I, a la Facultad de Ciencias Sociales de la Universidad Nacional José Faustino Sánchez Carrión \\ Intrinsic motivation and academic performance of ingresants $2016-\mathrm{I}$, to the Faculty of Social Sciences of the National University Jose Faustino Sanchez Carrion
}

\author{
Milagro Humberta Santiago Trujillo
}

\begin{abstract}
RESUMEN
Objetivo: Establecer la relación entre la motivación intrínseca y el rendimiento académico final de los ingresantes. Material y Métodos: Para esta investigación se utilizó el Test de motivación intrínseca de Alberto Vílchez Ortiz, con el Alfa de Cronbach (2016). Y para evaluar el rendimiento académico hemos utilizado las actas finales de notas del Semestre 2016 - I. Resultados: De un total de 126 ingresantes 2016 - I de la Facultad de Ciencias Sociales, que representan el 100\%; 66 (52,5\%) muestran excelente motivación intrínseca, de ellos 41 (32,5\%) presentan buen rendimiento académico y $24(19,2 \%)$ presentan aceptable rendimiento académico. Conclusión: Cuando la motivación de los estudiantes ingresantes es mejor, hay una tendencia muy alta a que tengan un mejor rendimiento académico.
\end{abstract}

Palabras claves: Motivación intrínseca, rendimiento académico, ingresantes, estimulación, filiación, empoderamiento.

\begin{abstract}
Objective: To establish the relationship between intrinsic motivation and the final academic performance of the entrants. Material and Methods: For this investigation, the Alberto Vílchez Ortiz Intrinsic Motivation Test was used, with Cronbach's Alpha (2016). And to evaluate the academic performance we have used the final minutes of the Semester 2016 - I. Results: Of a total of 126 entries 2016 - I of the Faculty of Social Sciences, which represent $100 \%$; 66 (52.5\%) show excellent intrinsic motivation, 41 of them (32.5\%) have good academic performance and 24 (19.2\%) have acceptable academic performance. Conclusion: When the motivation of incoming students is better, there is a very high tendency for them to have a better academic performance.
\end{abstract}

Keywords: Intrinsic motivation, academic performance, entrants, stimulation, affiliation, empowerment.

\section{INTRODUCCIÓN}

Este trabajo de investigación, procura hacer un diagnóstico especifico de como los ingresantes a las tres Escuelas pertenecientes a la Facultad de Ciencias Sociales manifiestan su motivación intrínseca, es decir de como vienen ellos preparados interiormente a la universidad.

Si el aprendizaje es multifactorial, ya que depende de diferentes componentes intrapersonales además contextuales. Dentro de esa variedad de factores interindividuales se encuentra la normalidad del sistema nervioso de cada quien, la integridad de los sentidos de cada quien, así como también los factores emocionales variados y variables que cada estudiante desarrolla en el curso de sus vidas.

Recalcando también algo que lleva a que una persona persiga el logro de lo que se ha propuesto en diferentes aspectos, es esa energía que conlleva y motiva a la persona a cumplir con su tarea se llama motivación intrínseca, esto funciona en su aprendizaje personal desde su niñez, cruzando por la adolescencia y llegando a la adultez.

Haciendo una descripción de la problemática en el ámbito local, los estudiantes de educación superior no participan con motivación ante los contenidos propios de su área. Es común que los alumnos no cumplan con las asignaciones previstas por el docente, en muchas sesiones de clases el docente les asignaba los trabajos de investigación para que lo vayan desarrollando progresivamente en casa, o en todo caso les asignaba trabajos monográficos o resúmenes en donde el docente esperaba trabajarlas en la próxima sesión, pero un gran porcentaje de alumnos no las realizaba con el tiempo que requiere una tarea universitaria.

Esto ocasionaba que el docente tendría que hacer una retroalimentación de toda la clase repasando lo previsto anteriormente. Recalca que continuamente los alumnos hacen actividades diferentes a las que se proponen en clases y la preocupación aumenta aún más cuando vemos alumnos con carencias de motivación por aprender ocasionando así, deficiencia en el aprendizaje significativo del alumnado. Esta es una responsabilidad integrada del entorno familiar y social del alumno, es decir, la falta de seguridad y responsabilidad del entorno del estudiante que lo aparta de esa fortaleza que requiere para adquirir nuevos aprendizajes.

En base a lo anterior se planteó la siguiente pregunta general: ¿Qué relación hay entre la motivación intrínseca y el rendimiento académico de los ingresantes 2016-I, a la Facultad de Ciencias Sociales de la Universidad Nacional José Faustino Sánchez Carrión de Huacho?

Se justifica, porque es un trabajo de investigación práctica provechosa, que ayudará a conocer de forma clara y directa como las variables motivación intrínseca y la del rendimiento académico, mantienen una relación en lo que se refiere al entorno del estudiante universitario. De considerar la investigación beneficiosa las autoridades correspondientes podrían tomar como referencia los resultados de esta investigación a fin de optimar algunos aspectos que pueda ayudar a mejorar el estado motivacional y como consecuencia mejorar su rendimiento académico.

(Vivar, 2013) determino la relación que existe entre la motivación para el aprendizaje y el rendimiento académico en el área e inglés de los estudiantes de primer grado de educación secundaria de la institución educativa Fe y Alegría N49 de Piura, 2012.

La población de estudio estuvo constituida por 54 alumnos del primer grado de educación secundaria y la docente del área de Inglés de la Institución Educativa en mención. Los resultados fueron los siguientes, el 59\% mostraba una baja motivación, así como también el $67 \%$ tenía un rendimiento entre bajo y muy bajo. El grado de correlación entre la motivación para el aprendizaje y el nivel de logro del criterio expresión y comprensión oral. Comprensión de textos, producción de textos y el criterio actitud del área de inglés, se presentan de muy baja a baja correlación.

Es así que tomamos como referencia estos conceptos para mejorar nuestras técnicas de aprendizaje. Las estrategias utilizadas van a poner un énfasis de importancia en la motivación; calificada como la base del éxito para alcanzar significancia en el aprendizaje y en el rendimiento académico. 


\section{OBJETIVOS}

\section{Objetivo general}

Establecer qué relación hay entre la motivación intrínseca y el rendimiento académico de los ingresantes 2016 - I a la Facultad de Ciencias Sociales de la Universidad Nacional José Faustino Sánchez Carrión de Huacho.

\section{MATERIALES Y MÉTODOS}

El diseño metodológico es descriptivo correlacional no experimental, porque se realizará si manipular deliberadamente ninguna de las variables y después analizarlos.

Es una investigación de corte trasversal porque los datos se recolectarán en un único momento.

El tipo de investigación es básica porque se realiza con conocimientos y teorías de nivel descriptivo, correlacional considerando que se describirá y relacionará a las variables y sus dimensiones.

El enfoque de la investigación es cuantitativo debido a que se utilizará la recolección de datos y su posterior análisis para la demostración del establecimiento de los objetivos. (Hernández, Fernández y Baptista, 2003)

La Población a estudiar en esta investigación está integrada por los ingresantes 2016 - I, en su totalidad, 44 a las Escuelas de Trabajo Social, 42 a Sociología y 40 a Ciencias de la Comunicación. 92 de ellos son de sexo femenino y 34 de sexo masculino.

\section{Técnicas e instrumentos de recolección de datos a emplear}

Serán la observación no estructurada a los ingresantes 2016 I, la misma que se realizara en el momento de la aplicación del instrumento.

a. Variables relevantes.

- Independiente: Motivación intrínseca

- Dependiente: Rendimiento académico

b. Variables intervinientes

- Sexo: Femenino/Masculino

- Escuelas: T. Social / Sociología / CC. de la Comunicación.

Descripción de los instrumentos.

a. Para la Variable Independiente: Motivación intrínseca. - Para esta variable se utilizará el Test de Motivación intrínseca de Alberto Vílchez Ortiz que está estructurada con 40 preguntas y cuya calificación se realizará de la siguiente manera: Frecuente 2,5 puntos. A veces 2,0, Neutro 1,5. Casi nunca 1,0, Nunca 0,5.

b. Para la Variable Dependiente: Rendimiento académico. Para su evaluación utilizaremos las actas de cada curso o materia desarrollada de cada una de las Escuelas Profesionales en estudio de la Facultad de Ciencias Sociales; proporcionadas por la Oficina de Asuntos Académicos de la Universidad.

\section{Técnicas para el procesamiento de la información.}

Estaremos haciendo uso de las técnicas del programa de Word y de Excel a fin de elaborar las tablas y figuras correspondientes, y finalmente utilizaremos el coeficiente de correlación de Spearman para correlacionar las variables de la investigación, dado que estas tienen una medición de escala ordinal. La prueba consiste en determinar si hay correlación significativa entre las variables $\mathrm{X}$ e $\mathrm{Y}$, cuyo procedimiento es el siguiente:

$1^{\circ}$ Planteamiento de las hipótesis estadísticas al nivel de significación del $\alpha=0,05$ o 0,01:

Las variables no están correlacionadas

Las variables están correlacionadas

$2^{\circ}$ Resultados de la prueba de hipótesis

Serán obtenidos usando el software Estadístico SPSS versión 22

$3^{\circ}$ Decisión:

$4^{\circ} \mathrm{Si}$-valor es mayor que $\alpha=0.05$ o 0.01 , entonces, aceptamos la Hipótesis Nula. Caso contrario se rechaza.

\section{RESULTADOS}

Existe relación entre la motivación intrínseca y el rendimiento académico de los ingresantes 2016 - I a la Facultad de Ciencias Sociales de la Universidad Nacional José Faustino Sánchez Carrión, Huacho.

\section{Tabla 1}

Prueba de Hipótesis General

\begin{tabular}{|c|c|c|c|c|}
\hline \multicolumn{5}{|c|}{ Correlación de Spearman } \\
\hline & & \multicolumn{3}{|c|}{$\begin{array}{l}\text { Rendimiento } \\
\text { académico }\end{array}$} \\
\hline \multirow{3}{*}{$\begin{array}{l}\text { Rho de } \\
\text { Spearman }\end{array}$} & \multirow{3}{*}{$\begin{array}{l}\text { Motivación } \\
\text { intrínseca }\end{array}$} & $\begin{array}{l}\text { Coeficiente } \\
\text { de } \\
\text { correlación }\end{array}$ & 1,000 & Rsp $=0,322^{*}$ \\
\hline & & Sig. (bilateral) & & $P=0,000$ \\
\hline & & $\mathrm{N}$ & & 126 \\
\hline
\end{tabular}

El resultado de la significación bilateral es

$p$-valor $=0,000<a=0,05$, esto significa que hay evidencias suficientes para indicar que existe relación significativa entre la motivación intrínseca y el rendimiento académico de los 126 ingresantes 2016-I a la Facultad de Ciencias Sociales; siendo el grado de relación positiva y baja con un coeficiente de Rsp $=0,322$

\section{Prueba de hipótesis especificas:}

Existe relación entre la motivación intrínseca y el rendimiento académico de los ingresantes 2016-I a la Escuela Profesional de Trabajo Social de la Facultad de Ciencias Sociales de la Universidad Nacional José Faustino Sánchez Carrión, Huacho.

Tabla 2

Hipótesis especifica 01

\begin{tabular}{|c|c|c|c|c|}
\hline \multicolumn{5}{|c|}{ Correlación de Spearman } \\
\hline & & \multicolumn{3}{|c|}{$\begin{array}{c}\text { Rendimiento } \\
\text { académico }\end{array}$} \\
\hline \multirow{3}{*}{$\begin{array}{l}\text { Rho de } \\
\text { Spearman }\end{array}$} & \multirow{3}{*}{$\begin{array}{l}\text { Motivación } \\
\text { intrínseca }\end{array}$} & $\begin{array}{l}\text { Coeficiente } \\
\text { de } \\
\text { correlación }\end{array}$ & 1,000 & $\mathrm{Rsp}=0,316^{*}$ \\
\hline & & Sig. (bilateral) & & $P=0,047$ \\
\hline & & $\mathrm{N}$ & & 44 \\
\hline
\end{tabular}

El resultado de la significación bilateral es

$p$-valor $=0,047<a=0.05$, esto significa que hay evidencias suficientes para indicar que existe relación significativa entre la motivación intrínseca y el rendimiento académico de los 44 ingresantes 2016-I a la Escuela Profesional de Trabajo Social; siendo el grado de relación positiva y baja con un coeficiente de Rsp $=0,316$ 
Existe relación entre la motivación intrínseca y el rendimiento académico de los ingresantes 2016 - I a la Escuela Profesional de Sociología de la Facultad de Ciencias Sociales de la Universidad Nacional José Faustino Sánchez Carrión, Huacho.

Tabla 3

Hipótesis especifica 02

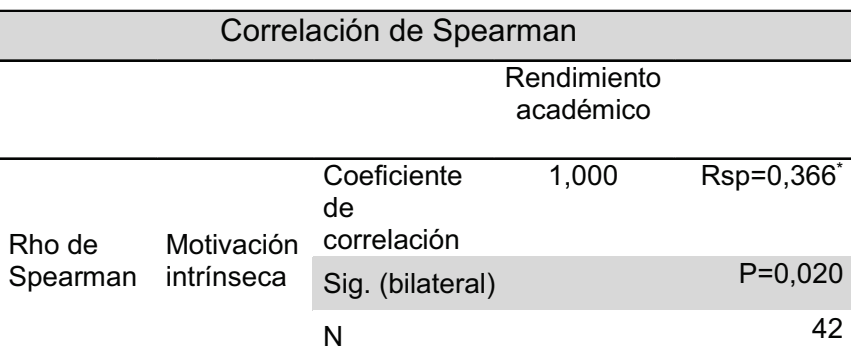

*La correlación es significativa en el nivel 0,05 (bilateral)

El resultado de la significación bilateral es

$p$-valor $=0,020<a=0.05$, esto significa que hay evidencias suficientes para indicar que existe relación significativa entre la motivación intrínseca y el rendimiento académico de los 42 ingresantes 2016-I a la Escuela Profesional de Sociología; siendo el grado de relación positiva y baja con un coeficiente de $\mathrm{Rsp}=0,366$.

Existe relación entre la motivación intrínseca y el rendimiento académico de los ingresantes 2016 - I a la Escuela Profesional de Ciencias de la Comunicación de la Facultad de Ciencias Sociales de la Universidad Nacional José Faustino Sánchez Carrión, Huacho.

Tabla 4

Hipótesis especifica 03

\begin{tabular}{|c|c|c|c|c|}
\hline \multicolumn{5}{|c|}{ Correlación de Spearman } \\
\hline & & \multicolumn{3}{|c|}{$\begin{array}{c}\text { Rendimiento } \\
\text { académico }\end{array}$} \\
\hline \multirow{3}{*}{$\begin{array}{l}\text { Rho de } \\
\text { Spearman }\end{array}$} & \multirow{3}{*}{$\begin{array}{l}\text { Motivación } \\
\text { intrínseca }\end{array}$} & $\begin{array}{l}\text { Coeficiente } \\
\text { de } \\
\text { correlación }\end{array}$ & 1,000 & $\mathrm{Rsp}=0,336^{*}$ \\
\hline & & Sig. (bilateral) & & $P=0,034$ \\
\hline & & $\mathrm{N}$ & & 40 \\
\hline
\end{tabular}

*La correlación es significativa en el nivel 0,05 (bilateral)

El resultado de la significación bilateral es

$\mathrm{p}$-valor $=0,034<\mathrm{a}=0,05$, esto significa que hay evidencias suficientes para indicar que existe relación significativa entre la motivación intrínseca y el rendimiento académico de los 40 ingresantes 2016-I a la Escuela Profesional de Ciencias de la Comunicación; siendo el grado de relación positiva y baja con un coeficiente de $\mathrm{Rsp}=0,336$.

\section{DISCUSIÓN}

Los resultados obtenidos en la investigación tiene similitud a lo hallado por (Martínez, 2011), quien reporta que el $74 \%$ de los estudiantes en críticas para la profesión y para la vida, que además es esencial afirmando que una de las competencias para aprender, es la competencia de auto-motivación presentaban muy buena automotivación, logrando también que el $64 \%$ tenian un rendimiento académico bueno y muy bueno. Lo que significa que cuando el en estudiante existe una mejor motivación, mejor será también el rendimiento académico de los mismos. (pág. 83).

Del total de 126 ingresantes 2016 - I de la Facultad de Ciencias Sociales el $52.5 \%$ muestran excelente motivación intrínseca, de ellos el $32.5 \%$ presentan buen rendimiento académico y el
19.2\% aceptable rendimiento académico; así mismo el 35\% muestran buena motivación intrínseca, de ellos el 19.2\% presentan buen rendimiento académico y el $15 \%$ aceptable rendimiento académico; en el mismo sentido, el $12.5 \%$ muestran regular motivación intrínseca y de ellos el $5 \%$ y el $5.8 \%$ presentan regular y un deficiente rendimiento académico respectivamente.

De 44 de los ingresantes 2016 - I de la Escuela Profesional de Trabajo Social, el $47.5 \%$ muestran excelente motivación intrínseca, de ellos el $35 \%$ presentan buen rendimiento académico y $12.5 \%$ aceptable rendimiento académico; asimismo, el $42.5 \%$ muestran buena motivación intrínseca, de ellos el $20 \%$ presentan buen rendimiento académico y el $20 \%$ aceptable rendimiento académico; por otro lado, el $7.5 \%$ muestran regular motivación intrínseca y un deficiente rendimiento académico.

De 42 de los ingresantes 2016 - I a la Escuela Profesional de Sociología el $57.5 \%$ muestran excelente motivación intrínseca, de ellos el $35 \%$ presentan buen rendimiento académico y el $20 \%$ aceptable rendimiento académico; así mismo el $35 \%$ muestran buena motivación intrínseca, de ellos el $(22.5 \%$ presentan buen rendimiento académico y el $12.5 \%$ aceptable rendimiento académico; por otro lado el $7.5 \%$ muestran regular motivación intrínseca y un deficiente rendimiento académico.

De 40 de los ingresantes 2016 - I a la Escuela Profesional de Ciencias de la Comunicación, 52.5\% muestran excelente motivación intrínseca, de ellos el $27.5 \%$ presentan buen rendimiento académico y el $25 \%$ presentan aceptable rendimiento académico; así mismo, el $27.5 \%$ muestran buena motivación intrínseca, de ellos el $15 \%$ presentan buen rendimiento académico y $12,5 \%$ presentan aceptable rendimiento académico; por otro lado, $15 \%$ muestran regular motivación intrínseca y un aceptable rendimiento académico.

\section{CONCLUSIÓN}

Cuando la motivación de los estudiantes ingresantes es mejor, hay una tendencia muy alta a que tengan un mejor rendimiento académico.

\section{AGRADECIMIENTO}

Al Dr. Hugo Rojas Carranza por su amistad, tiempo, paciencia y haberme brindado sus sabios conocimientos durante todo este tiempo. A mi padre, porque sé que estás aquí. A Daniel por ser mi compañero eterno. A mis hijos por ser mi motor.

\section{REFERENCIAS BIBLIOGRÁFICAS}

Hernández, Fernández y Baptista. (2003). Enfoque cuantitativo en las investigaciones, p.58-270-63-64. Obtenido de http://catarina.udlap.mx/u_dl_a/tales/documentos/lad/bla nco_s_nl/capitulo3.pdf

Martínez, J. (2011). Automotivación y Rendimiento Académico en el Espacio Europeo de Educación Superior. Obtenido de http://www.eumed.net/rev/ced/28/jamg.htm

Vivar, M. (2013). La Motivación para el Aprendizaje y su Relación con el Rendimiento Académico en el área de Inglés de los Estudiantes del Primer Grado de Educación Secundaria. ( Tesis de Maestría en Educación con Mención en Teorías y Práctica Educativa). Obtenido de https://pirhua.udep.edu.pe/bitstream/handle/11042/1813/ MAE_EDUC_105.pdf?sequence=1 